\title{
Amyloid Plaque Imaging with a Targeted MRI Contrast Agent in a Transgenic Mouse Model of Alzheimer's Disease
}

\author{
Yongjie Xiong', Yi Qu', Zhe Min', Jun Wu $\mathbb{D}^{2}$, Suming Zhang', Zheng Xue' \\ 'Department of Neurology, Tongji Hospital, Tongji Medical College, Huazhong University of Science and Technology, Wuhan, Hubei, 430030, People's \\ Republic of China; ${ }^{2}$ Department of Neurology, The First Affiliated Hospital of Zhengzhou University, Zhengzhou, Henan, 450000, People's Republic \\ Of China
}

Correspondence: Zheng Xue, Department of Neurology, Tongji Hospital, Tongji College of Medicine, Huazhong University of Science and Technology, Wuhan, 430000, People's Republic of China, Tel +86 I 404234 5433, Email xuezheng@hust.edu.cn

Purpose: Magnetic resonance imaging (MRI) is a promising technique for detecting amyloid beta (A $\beta$ ) deposits in Alzheimer's disease $(\mathrm{AD})$. Contrast agents can reduce the scanning time and provide specific images to identify various structures. This study aimed to develop an ultrasmall superparamagnetic iron oxide (USPIO) nanoparticle coupled with a functional protein as a targeted MRI contrast agent to detect $\mathrm{A} \beta$ deposits.

Methods: The targeted MRI contrast agent USPIO nanoparticle was coupled with $\mathrm{A} \beta_{(16-20)}(\mathrm{KLVFF})$ and HIV-1 trans-activating transcriptor-protein transduction domain (Tat-PTD) to produce the Tat-PTD-USPIO-A $\beta_{(16-20)}$ structure to better penetrate the blood-brain barrier (BBB). In vitro studies were conducted to measure the coupling efficiency, including bicinchoninic acid protein assays, potassium ferrocyanide staining of cardiac microvascular endothelial cells, 1.5T MRI, and toxicity assessments. The contrast agent was injected into five transgenic mice and five age-matched wild-type mice for MRI and histological studies. The targeted agent was diluted, and all mice were scanned with a 7.0 Tesla MRI-scanner. The brain tissues were processed as frozen sections for Thioflavin-S staining to detect A $\beta$.

Results: The coupling efficiency reached $97.75 \%$. The developed MRI contrast agent successfully passed through biological membranes, bound to $A \beta$ deposits, and decreased the MRI signal of tissue in vitro and in vivo. Compared with $A \beta_{(16-20)}$ or $\mathrm{A} \beta_{(1-40)}$ alone, there was no significant toxicity of Tat-PTD or $\mathrm{A} \beta_{(16-20)}$ when coupled with USPIO nanoparticles.

Conclusion: Our novel MRI contrast agent USPIO nanoparticle coupled with functional protein can pass through biological membranes in vitro and visualize $\mathrm{A} \beta$ deposits in $\mathrm{AD}$ animal models by MRI.

Keywords: Alzheimer's disease, transgenic mice, contrast agent, magnetic resonance imaging, nanomolecular imaging

\section{Introduction}

Alzheimer's disease (AD) is a neurodegenerative disease characterized by chronic progressive dementia, and its hallmark pathological changes include amyloid beta $(\mathrm{A} \beta)$ plaques, intracellular neurofibrillary tangles, and neuronal loss. ${ }^{1}$ The clinical diagnostic criteria of AD mainly rely on symptoms, scales, and imaging findings (eg encephalatrophy and hippocampal atrophy), and more accurate clinical diagnostic methods are urgently needed. Early diagnosis will become more important as $\mathrm{AD}$ treatments targeting specific molecular abnormalities become available. Pathogenetic studies have shown that $A \beta$ peptide accumulation in the brain is an early and central event, ${ }^{2}$ therefore $A \beta$ deposit detection is a promising technique to specifically diagnose the initial stage of $\mathrm{AD}$ when therapeutic interventions can be used to eliminate amyloid deposits.

Positron emission tomography has been employed to visualize $\mathrm{A} \beta$ plaques in the brain for the diagnosis of $\mathrm{AD},{ }^{3}$ however it has obvious shortcomings, such as radioligand use, limited spatial resolution and higher cost. ${ }^{4}$ These weaknesses can be overcome with magnetic resonance imaging (MRI). ${ }^{5}$ Models of AD such as transgenic mice can also be used for in vivo studies under high-field MRI. ${ }^{6}$ Although senile plaques have been observed with MRI in both 
postmortem human tissues and $\mathrm{AD}$ transgenic mice, ${ }^{7}$ better contrast agents are still urgently needed. The identification of $\mathrm{A} \beta$ without contrast agents is based on the chemical constituents (mainly iron) that affect the MRI signal. Unfortunately only a fraction of mature plaques contain sufficient iron, and the long scanning time is not appropriate for in vivo studies. ${ }^{8}$ Contrast agents can increase the sensitivity, particularly for smaller $A \beta$ deposits, while also shortening the imaging time. They can also provide specific imaging to differentiate $A \beta$ from other iron-containing structures, such as old hemorrhages and calcification.

Superparamagnetic iron oxide (SPIO) nanoparticles are an MRI contrast agent that can increase the magnetic sensitivity by decreasing the tissue relaxation time and providing real-time images to recognize biological processes at molecular and cellular levels. ${ }^{9}$ The ultrasmall SPIO (USPIO) nanoparticles used in the present study were functionalized with an amino-group (amino-USPIO), so they could be easily coupled with functional protein. ${ }^{10}$ Our preliminary work showed that USPIO nanoparticles coupled with $A \beta_{(1-40)}$ selectively labeled the senile plaques in the brain tissues of amyloid precursor protein/presenilin 1 (APP/PSI) transgenic mice, and were detected with 7.0 Tesla MRI. ${ }^{11}$ In this study, $\mathrm{A} \beta_{(16-20)}(\mathrm{KLVFF})$ was coupled as a probe to bind $\mathrm{A} \beta$ deposits, which was the smallest fragment that bound to the fulllength $A \beta$ protein. Studies have shown that the binding affinity of $A \beta_{(16-20)}$ is stronger than that of full-length $A \beta$, which prevents its inclusion into deposits. ${ }^{12}$ Moreover, the absence of $A \beta_{(25-35)}$ may decrease contrast agent toxicity. ${ }^{13}$ To enhance access through the brain-blood barrier (BBB), we coupled amino-USPIO with the HIV-1 trans-activating transcriptor-protein transduction domain (Tat-PTD). Tat-peptide is one of the most widely used molecules in cellular drug delivery systems. ${ }^{14}$ This region consists of 9-16 amino acids that can pass through biological membranes including the BBB. ${ }^{15}$ The present study was designed to develop USPIO nanoparticles coupled with Tat-PTD as a targeted MRI contrast agent to detect brain $A \beta$ deposits in vivo.

\section{Materials and Methods}

\section{Amino-USPIO Nanoparticles Coupled with Protein}

Amino-USPIO nanoparticles were provided by the School of Physics, HUST. ${ }^{10,16}$ The process of protein binding to amino-USPIO nanoparticles was as follows. First, $100 \mu \mathrm{L}$ of $0.2 \mathrm{~mol} / \mathrm{L}$ EDC $\cdot H C L$ [1-Ethyl-3-(3-dimethylaminopropyl) carbodiimide hydrochloride], $100 \mu \mathrm{L}$ of $0.02 \mathrm{~mol} / \mathrm{L}$ NHS (1-hydroxy-2, 5-pyrrolidinedione), $1 \mathrm{mg}$ Tat PTD, and/or $1 \mathrm{mg}$ $\mathrm{A} \beta$ dissolved in ammonia water were mixed with $1 \mathrm{~mL}$ amino-USPIO. The obtained solution was shaken for 30 min and allowed to react overnight at $4^{\circ} \mathrm{C}$, then the unreacted components were removed by ultrafiltration. Later, the products in the inserts of ultrafiltration tubes were the MRI contrast agents in aqueous phase, and the flow-through was used to measure the protein coupling efficiency with bicinchoninic acid (BCA) protein assay kits. This method can quantitatively measure solution levels by comparing optical densities (ODs) between unknown and known proteins. ${ }^{17}$

\section{In vitro Penetration Studies}

Cerebral microvascular endothelial cells (CMECs) were obtained from primary culture and incubated with $0.45 \mu \mathrm{mol} \mathrm{Fe} /$ $\mathrm{mL}$ FITC-Tat-PTD-USPIO-A $\beta_{(16-20)}$ under normal cell culture conditions for $48 \mathrm{~h}$. Afterwards, cells were observed under an inverted fluorescence microscope equipped with an Olympus CCD (Tokyo, Japan) and subsequently stained with potassium ferrocyanide.

CMECs were randomly divided into two groups for 48 -h incubation with or without Tat-PTD-USPIO-A $\beta_{(16-20)}$. Cells in both groups were washed with phosphate-buffered saline (PBS), collected into Eppendorf tubes, centrifuged at $1000 \mathrm{rpm}$ for $5 \mathrm{~min}$, and imaged with the 3.0T MRI scanner. The scanning parameters were as follows: T1 sequence (repetition time $[\mathrm{TR}]=540 \mathrm{~ms}$, echo time $[\mathrm{TE}]=6.0 \mathrm{~ms}$, field of view $[\mathrm{FOV}]=18 \times 18 \mathrm{~cm}$, matrix $=256 \times 192$, number of excitations $[\mathrm{NEX}]=2.00)$ and $\mathrm{T} 2$ sequence $(\mathrm{TR}=3000 \mathrm{~ms}, \mathrm{TE}=122.0 \mathrm{~ms}, \mathrm{FOV}=18 \times 14.4 \mathrm{~cm}$, matrix $=256 \times 192$, NEX $=8.00$ ). Equivalent volumes of pure contrast agent and unused culture medium of CMECs in tubes were used as the positive and blank controls, respectively. 


\section{In vitro Contrast Agent Toxicity Studies}

Contrast agent toxicities were compared for Tat-PTD-USPIO-A $\beta_{(16-20)}$, Tat-PTD-USPIO-A $\beta_{(1-40)}$ and Tat-PTD-USPIO using the MTT [3-(4, 5-dimethylthiazol-2-yl)-2, 5-diphenyl tetrazolium bromide] assay kits. Briefly, CMECs were added to the 96-well plates and randomly divided into seven groups: (1) $\mathrm{A} \beta_{(1-40)}$, (2) $\mathrm{A} \beta_{(16-20)}$, (3) Tat-PTD-USPIO-A $\beta_{(16-20)}$, (4) Tat-PTD-USPIO-A $\beta_{(1-40)}$, (5) Tat-PTD-USPIO, (6) USPIO, and (7) without treatment as the control group. After 72-h incubation, $10 \%$ MTT was added for another $4 \mathrm{~h}$ at $37^{\circ} \mathrm{C}$. After carefully removing the supernatant, the purple precipitate in each well was dissolved in $150 \mu \mathrm{L}$ dimethyl sulfoxide and the OD at $490 \mathrm{~nm}$ was measured using an iMark Microplate Absorbance Reader (Bio-Rad, Hercules, CA, USA). The concentration of $\mathrm{A} \beta_{(1-40)}$ was $20 \mu \mathrm{M}$, which was previously shown to be toxic to CMECs. ${ }^{18}$ Accordingly, the concentrations of $A \beta_{(16-20)}$ and bound $A \beta_{(1-40)} / A \beta_{(16-20)}$ in the Tat-PTD -USPIO-A $\beta_{(1-40)} /$ Tat-PTD-USPIO-A $\beta_{(16-20)}$ groups were also set at $20 \mu \mathrm{M}$. The concentrations of USPIO nanoparticles in the media of cells treated with Tat-PTD-USPIO and USPIO alone were matched to those of cells treated with Tat-PTD -USPIO-A $\beta_{(1-40)} /$ Tat-PTD-USPIO-A $\beta_{(16-20)}$.

\section{The Transgenic Mouse Model}

Five $A P P / P S 1$ transgenic mice served as the test group, whereas five age-matched wild-type $(\mathrm{C} 57 \mathrm{Bl} / 6 \mathrm{~J})$ mice were used as the control group. The model overexpresses the mutated forms of APP (APP695) and PS1, and the genotype of each mouse was confirmed by polymerase chain reaction analysis of mouse tail DNA. We observed abundant A $\beta$ deposits in the hippocampus and cortex of the 9-month-old mice, while occasional deposits were found in mice aged 6 months. In this study, 6-to-8-month-old mice were selected to imitate the early stage of AD. All animal experiments were approved by the Institutional Animal Care and Use Committee of Tongji Medical College, Huazhong University of Science and Technology according to the Guidance Suggestions for the Care and Use of Laboratory Animals.

\section{Contrast Agent Injection in vivo}

Tat-PTD-USPIO-A $\beta_{(16-20)}$ was diluted in PBS at a dose of $0.1 \mathrm{mmol} \mathrm{Fe} / \mathrm{kg}$ and infused via the caudal vein at a rate of 10 $\mathrm{ul} / \mathrm{s}$. The same infusion dose was repeated $24 \mathrm{~h}$ later.

\section{In vivo MRI Scanning of Transgenic Mice and Histological Studies}

$\mathrm{A} \beta_{(16-20)}$ coupled with HiLyte Fluor 555 was used to prepare the contrast agent [Tat-PTD-USPIO-A $\beta_{(16-20)}$-HiLyte Fluor 555], which exhibited red fluorescence so it could be differentiated from green fluorescence emitted by Thioflavin-S. All mice were scanned with a $7.0 \mathrm{~T}$ MRI scanner $2 \mathrm{~h}$ after the second contrast agent injection. To perform in vivo imaging, the mice were anesthetized with $2.5 \%$ isoflurane contained within $75 \% \mathrm{~N}_{2}$ and $21 \% \mathrm{O}_{2}$, while $1 \%$ isoflurane was used for maintenance anesthesia. The T2 sequence scanning parameters were as follows: TR $=3000.0 \mathrm{~ms}$, TE $=40.0 \mathrm{~ms}$, flip angle $=180.0 \mathrm{deg}, \mathrm{FOV}=2.02 \times 2.02 \mathrm{~cm}$, thickness $=0.5$, matrix $=256 \times 256$, and NEX $=12.00$.

After scanning, the brain tissues of all mice were sliced into frozen sections. The slices were sectioned continuously at a thickness of 10-20 $\mu \mathrm{m}$ with a cryostat, and two adjacent slices were categorized as one group. Then, the acquired sections were stained with Thioflavin-S, which specifically bind the senile plaques of AD. The stained sections were observed under an inverted fluorescence microscope equipped with an Olympus CCD to identify red and green fluorescence. The controls were selected according to the relative MR images.

\section{In vivo Binding Affinity of the Contrast Agent}

Unstained frozen brain sections of transgenic mice were incubated with FITC-Tat-PTD-USPIO-A $\beta_{(16-20)}$ at room temperature for $1 \mathrm{~h}$ and then washed with PBS. Frozen brain sections of wild-type mice were used as negative controls. Both sections were observed under the inverted fluorescence microscope equipped with an Olympus CCD to detect green fluorescence. 


\section{Statistical Analysis}

Data were analyzed with SPSS v. 13.0 software (SPSS Inc., Chicago, IL, USA). Statistical significance was analyzed by one-way analysis of variance and Student-Newman-Keuls tests. Differences were considered significant at $P<0.05$. All data were obtained from at least three independent experiments. Images were imported into Adobe Photoshop v. 7.0 (Adobe Systems, San Jose, CA, USA) for analysis.

\section{Results}

\section{Protein Characterization and Coupling Efficiency}

The magnetic nanoparticles in the aqueous phase obtained through the thermal decomposition technique were uniform and stable, with a core diameter of 6-10 $\mathrm{nm}$. The hydration radius of the sample in the aqueous phase was tested by laser particle size scattering analysis, which showed that the hydration radius of the sample was $\sim 50 \mathrm{~nm}$. In addition, the nanoparticles exhibited good dispersion in water, and no agglomeration phenomenon was found after 6 months. We also discovered that the nanoparticle surface was negatively charged at $-8.003 \mathrm{mV}$. The imaging capability on MRI for these nanomaterials was excellent, the $\mathrm{R}_{2}$ value of relaxation was $140 \mathrm{mmol}^{-1} \mathrm{sec}^{-1}$, and the property was stable after binding to $A \beta_{(16-20)}, A \beta_{(1-40)}$, and Tat-PTD proteins. All aspects of the tests met the requirements with acceptable biological stability in vitro.

Tat, $\mathrm{A} \beta_{(1-40)} / \mathrm{A} \beta_{(16-20)}$ and USPIO were connected at a ratio of 1:1:1. The coupling efficiencies of protein were determined to be $97.75 \%$ and $94.62 \%$ for Tat-PTD-USPIO-A $\beta_{(16-20)}$ and Tat-PTD-USPIO-A $\beta_{(1-40)}$, respectively, which were calculated by subtracting the initial protein from the protein in the flow-through with BCA protein assays.

\section{Biomembrane Penetration and Decreased T2 Relaxation Time}

The cells displayed green fluorescence under the inverted fluorescence microscope (Figure 1A) and were stained with potassium ferrocyanide (Figure 1B), suggesting that the cytoplasm contained iron oxide particles. The iron distribution was consistent with the fluorescence, indicating that the CMEC membranes were penetrated by FITC-Tat-PTD-USPIO$\mathrm{A} \beta_{(16-20)}$.

The in vitro MRI results are displayed in Figure 2. The liquids in tubes 1 and 4 were unused culture medium and pure contrast agent, respectively (Figure 2A: T1-weighted image; Figure 2B-F: T2-weighted image). The deposits in the bottoms of tubes 2 and 3 were CMECs incubated without and with Tat-PTD-USPIO-A $\beta_{(16-20)}$, respectively, and the appropriate amount of culture medium was added to match the volume. As exhibited by the images, tubes 1 and 2 were both bright, while tube 4 was dark. The bottom of tube 3 emitted a low signal, but the supernatant exhibited a high signal. These results indicated that CMECs incubated with Tat-PTD-USPIO-A $\beta_{(16-20)}$ showed the same low signal as that of the pure contrast agent; however, those incubated without Tat-PTD-USPIO-A $\beta_{(16-20)}$ presented the same high signal as that of culture medium, indicating that the contrast agents decreased the relaxation time of CMECs. Figure $2 \mathrm{C}-\mathrm{F}$ show the transverse images, which better depict the variation in MRI signals.

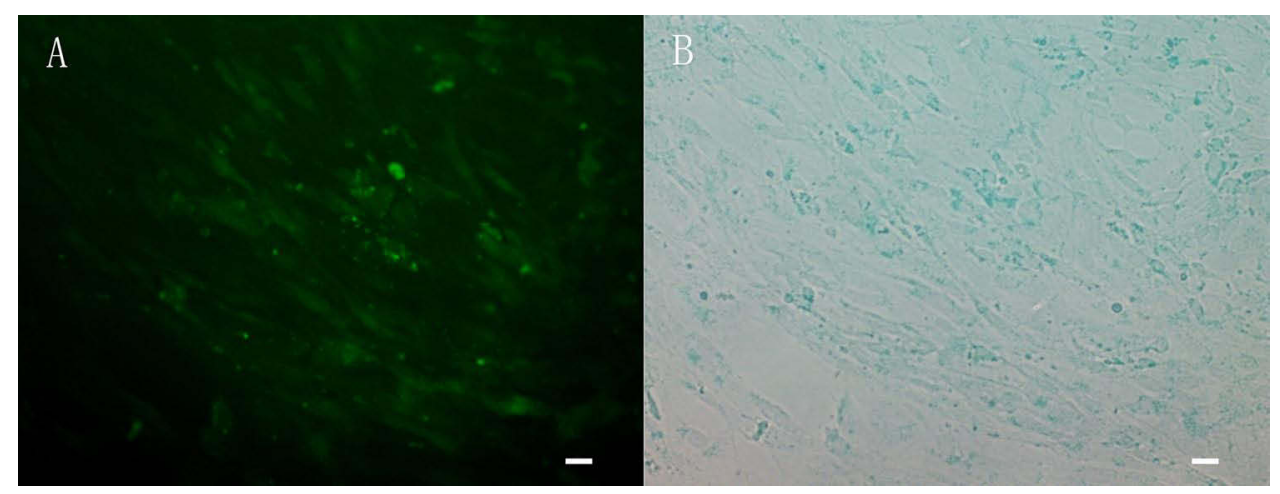

Figure I The cells showed green fluorescence $(\mathbf{A})$ and blue after staining by potassium ferrocyanide (B). The distribution of iron was consistent with the distribution of fluorescence, which indicated that the cell membrane of CMECs could be penetrated by FITC-Tat-PTD-USPIO-A $\beta_{(16-20)}$ 


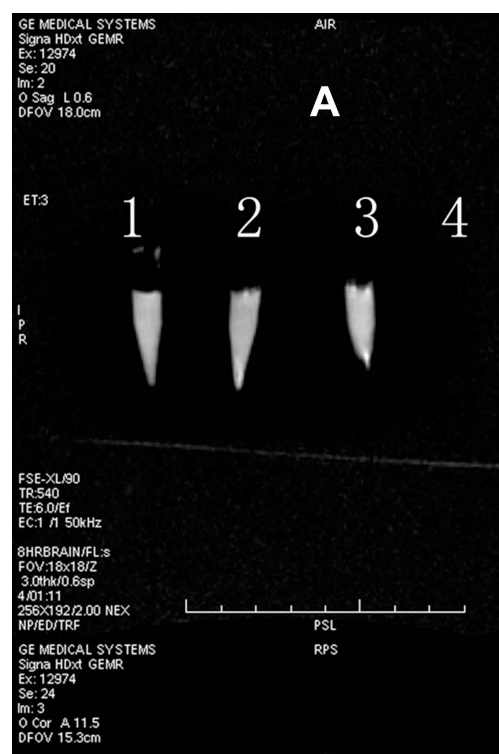

C

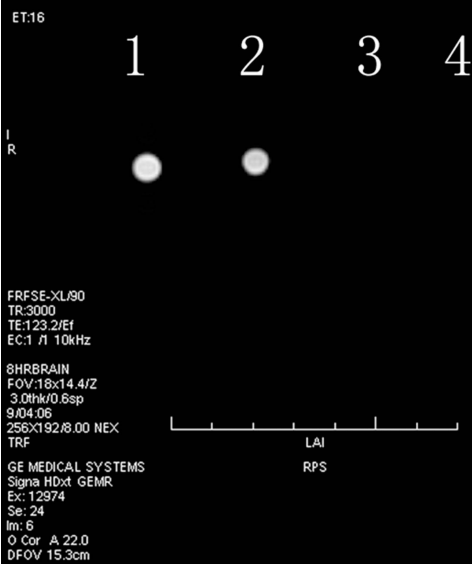

E
Tongli hospital GE MEDDCAL SYSTEMS
CMECS Signa DOXt GEMR

$\begin{array}{cc}\text { SY } & \text { EX: } 129 \\ \text { 20111219-SHENWEE } & \text { Se: } 16 \\ \text { Dec } 192011 & \mathrm{~m}: 2\end{array}$

$10.59: 08$ PM O O Sag $L 0.6$
Mag $=1.00$ OFOV $18.0 \mathrm{~cm}$

ROT: $90^{\circ}$
AIR

B
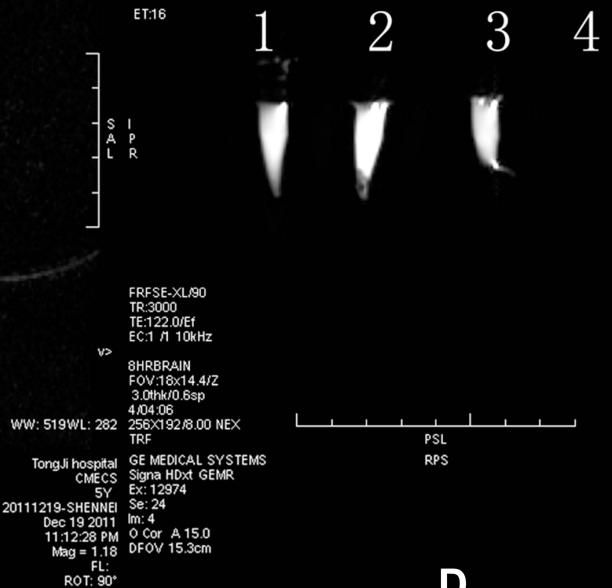

WW: 2056WL: 677

TongJi hospital
CMECS

20111219-SHEN

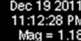

D

ROT: 90

ET:16

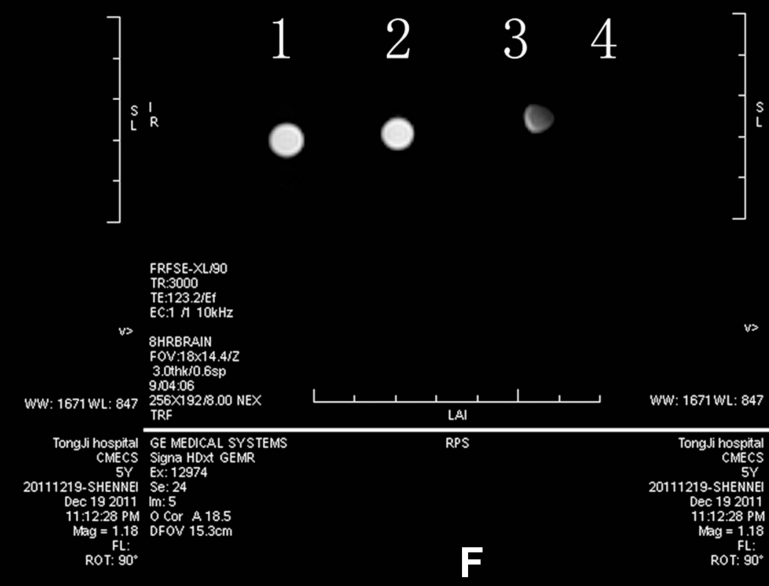

T:16

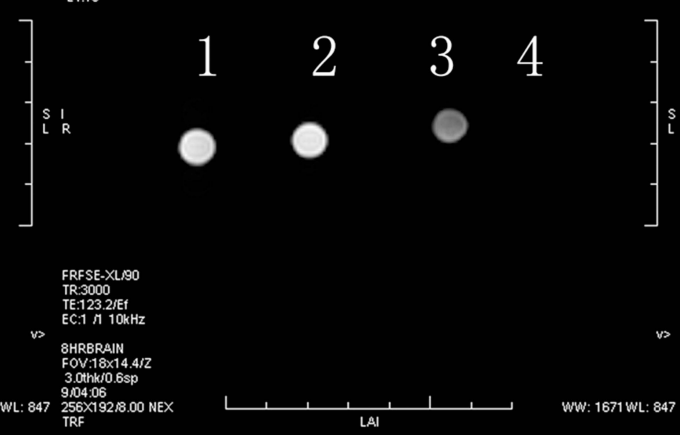

Figure 2 The MRI images of CMECs and FITC-Tat-PTD-USPIO-A $\beta_{(16-20)}$ incubated for $48 \mathrm{~h}$ in vitro. The tube I was unused culture medium. The deposits in the bottom of tube 2 and 3 were CMECs incubated without and with Tat-PTD-USPIO-A $\beta_{(16-20)}$, in which culture medium was added to match the volume. Tube 4 was the pure contrast agent. The results indicated that the MRI contrast agents could decrease the relaxation time of CMECs. There was a TI-weighted image (A), T2-weighted images (B-F), and cross-sectional images (C-F). The images were coronal scanning $(\mathbf{A}$ and $\mathbf{B})$ and plain scanning $(\mathbf{B}-\mathbf{F})$ of the four upright tubes. 


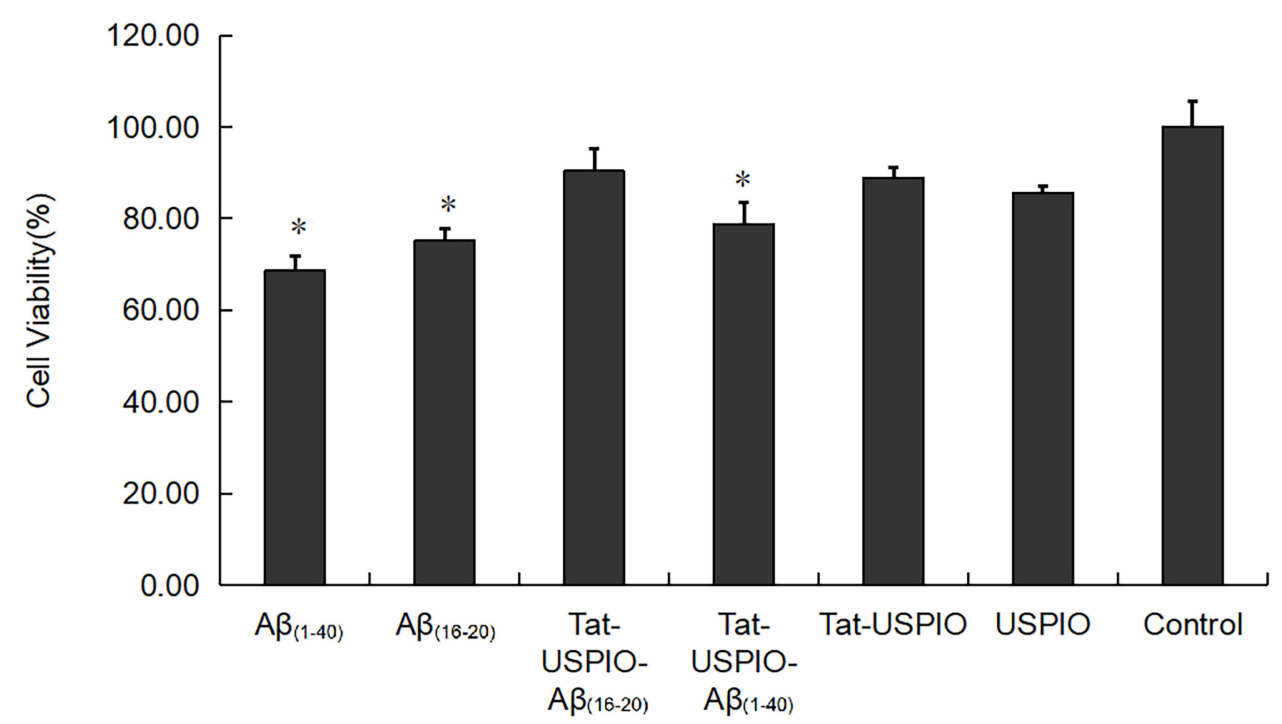

Figure 3 Histogram of toxicity test. Cell viability of untreated control cells is $100 \%$. $A \beta_{(I-40)}$ or $A \beta_{(16-20)}$ alone was toxic as expected ( $n=3$; $p<0.00 I$, $p<0.00 I$, respectively). There was no evidence that Tat-PTD and $A \beta_{(16-20)}$ coupled with USPIO have significant toxicity compared to the control group. Student-Newman-Keuls analysis showed the differences of $A \beta_{(I-40)}$ vs control, $A \beta_{(I-40)}$ vs USPIO, $A \beta_{(I-40)}$ vs Tat-PTD-USPIO, A $\beta_{(I-40)}$ vs Tat-PTD-USPIO-A $\beta_{(16-20)}$, $A \beta_{(16-20)}$ vs control, $A \beta_{(16-20)}$ vs Tat-PTD-USPIO, $A \beta_{(16-20)}$ vs Tat-PTD-USPIO-A $\beta_{(16-20)}$, Tat-PTD-USPIO-A $\beta_{(1-40)}$ vs control and USPIO vs control were all significant $(n=3$; all $P<0.05)$. Tat-PTD-USPIO-A $\beta_{(16-20)}$ vs control and Tat-PTD-USPIO vs control had no statistical difference. $* p<0.05$.

\section{Toxicity Comparison Among Contrast Agents}

As expected, $20 \mu \mathrm{MA} \beta_{(1-40)}$ or $\mathrm{A} \beta_{(16-20)}$ alone was toxic $(\mathrm{n}=3$; all $p<0.001)$. According to Student-Newman-Keuls analyses, the differences for $\mathrm{A} \beta_{(1-40)}$ vs control, $\mathrm{A} \beta_{(1-40)}$ vs USPIO, $\mathrm{A} \beta_{(1-40)}$ vs Tat-PTD-USPIO, $\mathrm{A} \beta_{(1-40)}$ vs Tat-PTDUSPIO-A $\beta_{(16-20)}, A \beta_{(16-20)}$ vs control, $A \beta_{(16-20)}$ vs Tat-PTD-USPIO, $A \beta_{(16-20)}$ vs Tat-PTD-USPIO-A $\beta_{(16-20)}$, Tat-PTDUSPIO-A $\beta_{(1-40)}$ vs Control, and USPIO vs control were all significant $(\mathrm{n}=3$; all $\mathrm{p}<0.05)$. However, there were no significant differences for Tat-PTD-USPIO-A $\beta_{(16-20)}$ vs control or Tat-PTD-USPIO vs control, so there was no evidence that Tat-PTD and $A \beta_{(16-20)}$ coupled with USPIO had significant toxicity compared with the control group. Instead, coupling decreased the toxicity relative to $A \beta_{(16-20)}$ or $A \beta_{(1-40)}$ alone (Figure 3).

\section{Matching in vivo MRI Findings and Histological Studies}

Tat-PTD-USPIO-A $\beta_{(16-20)}$-HiLyte Fluor 555 was used as the MRI contrast agent, so the red fluorescence corresponded to deposits of contrast agents, while the green fluorescence represented senile plaques stained with Thioflavin-S. The match between red and green fluorescence in one section indicating that the contrast agents bound to the senile plaques in vivo (Figure 4C and D). In addition, the distribution of dark spots in T2-weighted images was matched to that of red and green fluorescence, indicating that this MRI contrast agent bound to senile plaques in APP/PS1 transgenic mice and also decreased the MRI signals (Figure 4B), which was different from the imaging results of the control group.

\section{Specific Binding to Senile Plaques}

Green fluorescence spots on the frozen brain sections of transgenic mice corresponded to the senile plaque distribution (Figure 5A and B). There were no similar spots on frozen brain sections from wild-type mice (Figure 5C and D), suggesting that the contrast agents bound to the senile plaques in the brains of APP/PS1 transgenic mice.

\section{Discussion}

The results of present study demonstrated that the targeted MRI contrast agent developed from USPIO nanoparticles coupled with the functional Tat-PTD-USPIO-A $\beta_{(16-20)}$ protein passed through the BBB and bound to A $\beta$ deposits in AD animal models as reflected with in vivo MRI. Our contrast agent targeted to $A \beta$ deposits could facilitate the development 


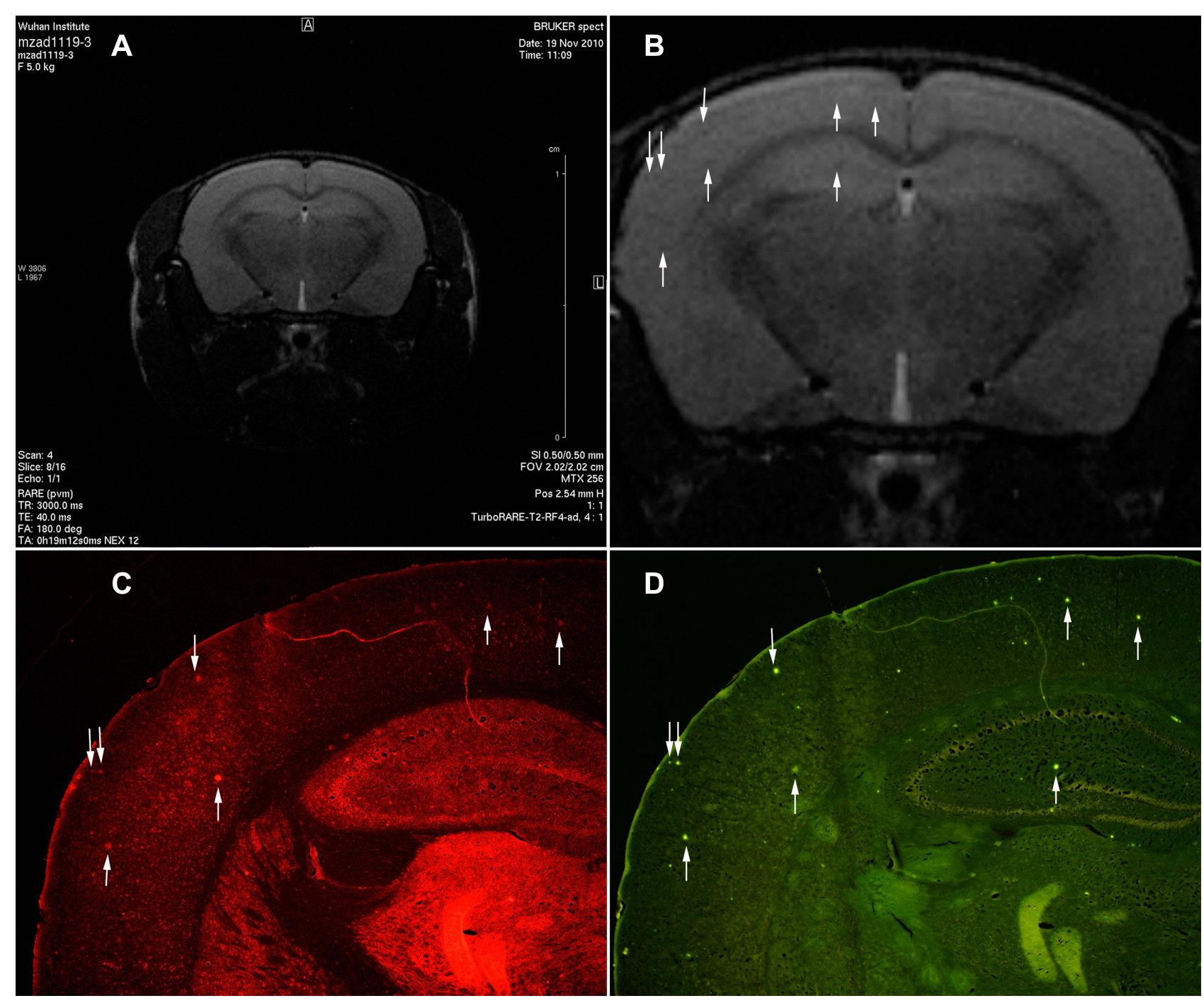

Figure 4 The transgenic mice injected Tat-PTD-USPIO-A $\beta_{(16-20)}$-HiLyte Fluor 555 were imaged with in vivo MRI to detect Amyloid plaques. It was the T2-weighted image (A) and partial enlarged (B). The red fluorescence referred deposits of the contrast agents in the frozen brain section (C), and the green fluorescence meant the senile plaques stained by Thioflavin-S (D). The match of the red and green fluorescence in one section manifested that the contrast agents could bind to senile plaques in vivo. The distribution of dark spots in the T2-weighted image matched to the distributions of red and green fluorescence, which indicated this MRI contrast agents could bind to the senile plaques of APPIPSI transgenic mice and also decreased the signal of them. The arrows indicated the contrast agents bounding to the senile plaques in vivo.

of in vivo diagnostic and monitoring techniques for AD progression. Further research and development of Tat-PTDUSPIO-A $\beta_{(16-20)}$ as a potential therapeutic intervention in AD animal models is also needed.

We developed USPIO coupled with Tat PTD and A $\beta$ according as an MRI contrast agent for AD. A $\beta_{(1-40)}$ and $\mathrm{A} \beta_{(16-20)}$ were used as the probes to bind senile plaques in our previous and present studies. Both probes detected $\mathrm{A} \beta$ deposits in the brain tissue of $A P P / P S 1$ transgenic mice when they were coupled with USPIO particles. As previously reported, the toxicity of $\mathrm{A} \beta$ at micromolar concentration was a potential stumbling block in a recent study. ${ }^{18}$ However, our cell toxicity tests showed no evidence that our MRI contrast agent had obvious toxicity compared with other contrast agents. More specifically, $A \beta_{(16-20)}$ had less toxicity than $A \beta_{(1-40)}$, especially when they were only one component of the MRI contrast agent. Among the different groups, cell viability in the Tat-PTD-USPIO-A $\beta_{(16-20)}$ group was the closest to that of control group, consistent with our hypothesis; this is the main reason why we prefer to use $A \beta_{(16-20)}$ over other developed MRI contrast agents. There are still other reasons considerations: the synthetic cost of $A \beta_{(16-20)}$ is much lower than that of $A \beta_{(1-40)}$, and the coupling efficiency of $A \beta_{(16-20)}$ with USPIO is higher than $A \beta_{(1-40)}$ due to the smaller molecular volume of $\mathrm{A} \beta_{(16-20)}$. 

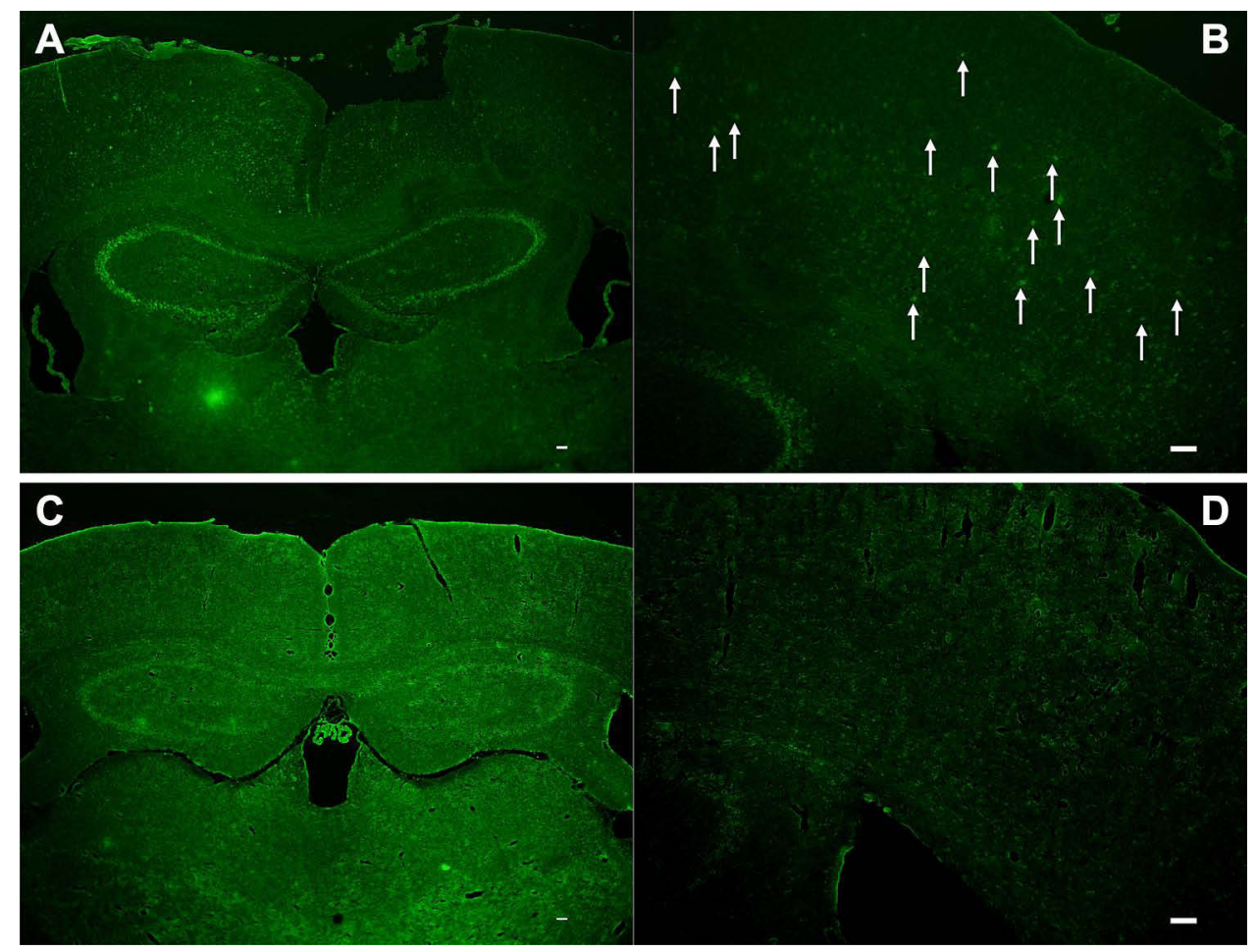

Figure 5 The frozen brain sections were incubated with FITC-Tat-PTD-USPIO-A $\beta_{(16-20)}$. The frozen brain sections of AD transgenic mice showed green fluorescent spots, and were similar with the distribution of senile plaques (A and $\mathbf{B})$. No similar spots were on the frozen brain sections of wild-type mice (C and $\mathbf{D})$. The arrows indicated the contrast agents bounding to the senile plaques in vivo.

The BBB is a dynamic and complex interface between the blood and central nervous system (CNS) parenchyma that protects the brain from toxic substances. Many drugs targeting the CNS are limited by the BBB. In our study, the TatPTD construct was used to facilitate entry of the MRI contrast agent past the BBB, similar to the reported ability of CNS delivery in a recent study. ${ }^{19}$ Therefore, transport via Tat-PTD is specific due to its coupling with the contrast agent is improved compared with a delivery system where the BBB is disrupted (eg mannitol injection), and it is associated with a lower risk of infection or other unknown factors caused by BBB opening.

In recent years, the use of USPIOs coupled with a targeted peptide bound to $A \beta$ as a method for the specific MRI detection of $A \beta$ plaques has been in great demand, and investigations have focused on discovering optimal targeted peptides. ${ }^{20-21}$ Here we employed PHO (C-IPLPFYN-C) or PHI (C-FRHMTEQ-C) due to their good A $\beta$ affinity. Some studies have co-injected intravenously with mannitol or polyethylene glycol to transiently open the BBB to amplify the abilities of USPIO coupled with $A \beta_{(1-42)}$ to detect $A \beta$ in $A D$ model mice. ${ }^{22-23}$ Our targeted agent has less toxicity and lower cost because of the absence of $A \beta_{(25-35)}$, and Tat-PTD can easily pass the BBB, so our contrast agent has lower risks compared with previous studies. Our results suggest that this novel targeted agent is more promising for detecting $\mathrm{A} \beta$ deposits and merits further exploration.

Several strengthens and limitations of our study should be noted. Firstly, our USPIO nanoparticles were watersoluble, biocompatible, non-toxic, appropriately sized, and presented a high relaxivity coefficient as an MRI contrast agent for in vivo imaging. Secondly, $A \beta_{(16-20)}$ had stronger binding affinity, lower toxicity, and lower production cost compared to $A \beta_{(1-40)}$. Moreover, it specifically facilitated contrast agent entry through the BBB with Tat-PTD, which reduced the risk of other unknown substances entering the CNS. Our results should also be considered in the context of several limitations. Firstly, the safety of Tat-PTD was not verified in human subjects since it was obtained from the HIV-1 construct. Secondly, quantitative experiments are needed in to explore the statistic differences. Lastly, the whole-body distribution of our MRI contrast agent should be assessed in the future studies. 


\section{Conclusion}

We developed a novel targeted MRI contrast agent Tat-PTD-USPIO-A $\beta_{(16-20)}$ to facilitate the diagnosis of AD and dynamically monitor $A \beta$ deposition. Further research is needed to assess the utility of Tat-PTD-USPIO-A $\beta_{(16-20)}$ as potential therapeutic interventions in $\mathrm{AD}$ animal models, and additional safety studies are needed before it can be used in humans.

\section{Acknowledgments}

This work was supported by The National High Technology Research and Development Program of China (Grant No 2007AA03Z312). The authors thank Dr Ming Ma and Dr Zuli Liu for generously providing the MRI contrast agent.

\section{Disclosure}

The authors report no conflicts of interest in this work.

\section{References}

1. Perl DP. Neuropathology of Alzheimer's disease. Mt Sinai J Med. 2010;77(1):32-42. doi:10.1002/msj.20157

2. Karran E, Mercken M, De Strooper B. The amyloid cascade hypothesis for Alzheimer's disease: an appraisal for the development of therapeutics. Nat Rev Drug Discov. 2011;10(9):698-712. doi:10.1038/nrd3505

3. Staffaroni AM, Elahi FM, McDermott D, et al. Neuroimaging in dementia. Semin Neurol. 2017;37(5):510-537. doi:10.1055/s-0037-1608808

4. Valotassiou V, Malamitsi J, Papatriantafyllou J, et al. SPECT and PET imaging in Alzheimer's disease. Ann Nucl Med. 2018;32(9):583-593. doi:10.1007/s12149-018-1292-6

5. Frisoni GB, Fox NC, Jack CR, Scheltens P, Thompson PM. The clinical use of structural MRI in Alzheimer disease. Nat Rev Neurol. 2010;6 (2):67-77. doi:10.1038/nrneurol.2009.215

6. Li MH, Wang PJ, Chen SQ, Zhang W, Gao XL. [The comparative study on high field MRI and pathology of Alzheimer's disease transgenic mice]. Zhonghua Yi Xue Za Zhi. 2011;91(13):876-879. Chinese.

7. Petiet A, Delatour B, Dhenain M. Models of neurodegenerative disease - Alzheimer's anatomical and amyloid plaque imaging. Methods Mol Biol. 2011;771:293-308.

8. Chamberlain R, Wengenack TM, Poduslo JF, Garwood M, Jack CR. Magnetic resonance imaging of amyloid plaques in transgenic mouse models of Alzheimer's disease. Curr Med Imaging Rev. 2011;7(1):3-7. doi:10.2174/157340511794653522

9. Mahmoudi M, Serpooshan V, Laurent S. Engineered nanoparticles for biomolecular imaging.. Nanoscale. 2011;3(8):3007-3026. doi:10.1039/ clnr10326a

10. Ma M, Zhan Y, Shen Y, Xia X, Zhang S, Liu Z. Synthesis of amino-group functionalized superparamagnetic iron oxide nanoparticles and applications as biomedical labeling probes. J Nanopart Res. 2011;13:3249-3257. doi:10.1007/s11051-011-0239-9

11. Zhan YQ, Wu J, Xu J, et al. [Study of specially labeling amyloid plaques in vivo in Alzheimer's transgenic mice with targeted magnetic nano-iron contrast agent]. Zhong Hua Shen Jing Ke Za Zhi. 2011;44:500-503. Chinese.

12. Tjernberg LO, Näslund J, Lindqvist F, et al. Arrest of beta-amyloid fibril formation by a pentapeptide ligand. J Biol Chem. 1996;271 (15):8545-8548. doi:10.1074/jbc.271.15.8545

13. Carvalho KM, França MS, Camarão GC, Ruchon AF. A new brain metalloendopeptidase which degrades the Alzheimer beta-amyloid 1-40 peptide producing soluble fragments without neurotoxic effects. Braz J Med Biol Res. 1997;30(10):1153-1156. doi:10.1590/S0100-879X1997001000002

14. Torchilin VP. Tat peptide-mediated intracellular delivery of pharmaceutical nanocarriers. Adv Drug Deliv Rev. 2008;60(4-5):548-558. doi:10.1016/ j.addr.2007.10.008

15. Rao K, Labhasetwar V. Trans-activating transcriptional activator (TAT) peptide-mediated brain drug delivery. J Biomed Nanotechnol. 2006;2:173-185. doi:10.1166/jbn.2006.036

16. Du GH, Liu ZL, Xia X, Jia LH, Zhang SM. Functionalization of Fe3O4 magnetic nanoparticles; 2006.

17. Smith PK, Krohn RI, Hermanson GT, et al. Measurement of protein using bicinchoninic acid. Anal Biochem. 1985;150(1):76-85. doi:10.1016/ 0003-2697(85)90442-7

18. Sadowski M, Pankiewicz J, Scholtzova H, et al. A synthetic peptide blocking the apolipoprotein $E / \beta$-amyloid binding mitigates $\beta$-amyloid toxicity and fibril formation in vitro and reduces $\beta$-amyloid plaques in transgenic mice. Am J Pathol. 2004;165(3):937-948. doi:10.1016/S0002-9440(10) 63355-X

19. Rao KS, Reddy MK, Horning JL, Labhasetwar V. TAT-conjugated nanoparticles for the CNS delivery of anti-HIV drugs. Biomaterials. 2008;29 (33):4429-4438. doi:10.1016/j.biomaterials.2008.08.004

20. Ansciaux E, Burtea C, Laurent S, et al. In vitro and in vivo characterization of several functionalized ultrasmall particles of iron oxide, vectorized against amyloid plaques and potentially able to cross the blood-brain barrier: toward earlier diagnosis of Alzheimer's disease by molecular imaging. Contrast Media Mol Imaging. 2015;10(3):211-224.

21. André S, Ansciaux E, Saidi E, et al. Validation by magnetic resonance imaging of the diagnostic potential of a heptapeptide-functionalized imaging probe targeted to amyloid- $\beta$ and able to cross the blood-brain barrier. J Alzheimers Dis. 2017;60(4):1547-1565. doi:10.3233/JAD-170563

22. Wadghiri YZ, Li J, Wang J, et al. Detection of amyloid plaques targeted by bifunctional USPIO in Alzheimer's disease transgenic mice using magnetic resonance microimaging. PLoS One. 2013;8(2):e57097. doi:10.1371/journal.pone.0057097

23. Yang J, Wadghiri YZ, Hoang DM, et al. Detection of amyloid plaques targeted by USPIO-A $\beta 1-42$ in Alzheimer's disease transgenic mice using magnetic resonance microimaging. Neuro Image. 2011;55(4):1600-1609. doi:10.1016/j.neuroimage.2011.01.023 


\section{Publish your work in this journal}

The International Journal of Nanomedicine is an international, peer-reviewed journal focusing on the application of nanotechnology in diagnostics, therapeutics, and drug delivery systems throughout the biomedical field. This journal is indexed on PubMed Central, MedLine, CAS, SciSearch ${ }^{\circledR}$, Current Contents ${ }^{\circledR} /$ Clinical Medicine, Journal Citation Reports/Science Edition, EMBase, Scopus and the Elsevier Bibliographic databases. The manuscript management system is completely online and includes a very quick and fair peer-review system, which is all easy to use. Visit http:// www.dovepress.com/testimonials.php to read real quotes from published authors.

Submit your manuscript here: https://www.dovepress.com/international-journal-of-nanomedicine-journal 